\title{
Research on micro compensation grinding method of helical groove for micro mill
}

\author{
HE Li-lun, LIU Zhi-bing, Lv Wei-wei, WANG Xi-bin, Jiao Li
}

( Beijing Institute of Technology, Beijing, 100081,China)

\begin{abstract}
Keywords: micro end mill; helical groove; micro compensation; grinding
\end{abstract}
\begin{abstract}
Micro tool manufacturing technology is the key for guaranteeing the manufacturing accuracy of micro milling cutter. According to the relative motion relationship and the meshing condition between the grinding wheel and the workpiece, the changes of normal vector of the intersection point for the grinding wheel generatrix was analyzed, and the equation of common contact line was established. Further, using the rotating cross-sectional shape formed by the rotation of contact line around the workpiece axis, the profile equation of the axial cross-sectional shape of the milling tool was deduced. During the process, a method of compensate deflection at $\mathrm{z}$ axis is proposed, through the calculation of bending deformation of the workpiece in grinding process. Experiments show that rake angle and core thickness of micro milling cutter obtained after the compensation, are good agreement with the simulation shape of the helical goove.
\end{abstract}

\section{Introduction}

The needs of micro tool with high cutting processing efficiency are more and more important in the aerospace system miniaturization.It is difficult for dressing the parts that feature size is in the range of $10 \mathrm{~m}$ to a high $1 \mathrm{~mm}$, so the service life of micro tool directly affects the performance of machining parts. Many scholars have tried to optimize the structure of micro tool, improve the surface quality of micro tool and seek ways of forming new improve performance of micro cutting tool. H. Ohmori ${ }^{[1]}$ studied the influence of surface quality of micro cutting tool on cutting tool fracture strength, analyzed different grinding wheel grain size how to effect the quality of micro cutting tool. H. Onikural ${ }^{[2]}$ studied the method of ultrasonic vibration grinding micro cylindrical cutter and fine flat drill, found the grinding force of ultrasonic vibration grinding of relatively low, which can reduce the damage of micro tool and get a higher ratio of length to diameter.Osamu Ohnishi ${ }^{[3-8]}$ studied many methods for grinding cylindrical linear edge of the micro milling cutter $(\phi 20) \mu \mathrm{m}$.But the works are mainly in the discussion around the grinding technological limit, rarely involved grinding structure shape precision problem.

When the micro rotary cutter diameter is less than $1 \mathrm{~mm}$, the low stiffness, radial force will make the micro tool relatively large bending deformation, the serious influence about accuracy problems of micro tool forming happen ,a method of micro milling cutter grinding to fine tune the amount of compensation is proposed.With the method,agrinding wheel contact line complete model is established to simulate spiral curved surface shape,according to the contact geometry condition of grinding wheel and workpiece.The model considers the flexural deformation.Through experiment, this method of micro compensation can effectively improve the forming precision of micro milling cutter.

\section{Axial spiral groove shape of grinding wheel}

\section{1 coordinates transform between the grinding wheel and the workpiece}

The coordinate system of the grinding wheel and the bar is shown in Fig 1, $\sigma=(\mathrm{O}: \mathrm{x}, \mathrm{y}, \mathrm{z})$ for a fixed coordinate system,its origin is located at $\mathrm{O}$, the rotary shaft as the $\mathrm{X}$ axis, the three axis direction unit vector which respectively with $i, j$ and $k$ representation; grinding wheel coordinate system for the sigma $\sigma 1=(01: x 1, y 1, z 1), x 1 a x i s$ for the grinding wheel axis, the unit direction of each axis vector respectively by $\mathrm{i} 1, \mathrm{j} 1$ and $\mathrm{k} 1 . \mathrm{M}$ is the contact point of the grinding wheel and 
the spiral groove, angle grinding wheel axis and the axis of the bar between the alpha and the center distance between the axis, y1 axis and y axis in the opposite direction, the homogeneous coordinate transformation between the two coordinate systems can be expressed as:

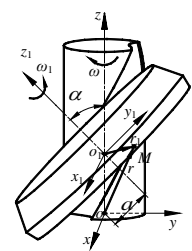

Fig 1 wheel and groove coordinate system

$$
\begin{aligned}
& r=\operatorname{Rot}(x, \pi) \cdot \operatorname{Rot}(y, \alpha) \cdot \operatorname{Trans}(0,-a, 0) \cdot r_{1} \\
& =\left[\begin{array}{cccc}
\cos \alpha & 0 & -\sin \alpha & 0 \\
0 & -1 & 0 & a \\
-\sin \alpha & 0 & -\cos \alpha & 0 \\
0 & 0 & 0 & 1
\end{array}\right] \cdot r_{1}
\end{aligned}
$$

\subsection{Contact wire wheel and groove surface}

Grinding wheel machining spiral groove exists inflection point, according to contact theory, grinding wheel groove was shown in Fig 2, there is always a contact wire of A-B-C-D-E-F, B-C and D-F segment is the touch line on the surface of the busbar rotary continuity. The A-B, E-F and C-D is the contact line formed inflection point and edgegrinding wheel place, in Fig 2b. The forming principles of the two kinds of contact line are different.Here are calculated respectively.

The wheel in the $\mathrm{x} 1-01-\mathrm{y} 1$ plane expressed as $\{\mathrm{x} 1, \mathrm{f}(\mathrm{x} 1), 0\}$, grinding wheel surface in the coordinates sigma 1 can be expressed as:

$$
r_{1}=\left\{x_{1} \quad f\left(x_{1}\right) \cos \varphi \quad f\left(x_{1}\right) \sin \varphi\right\}
$$

Where $\varphi$ indicates grinding wheel rotating around the $\mathrm{x} 1$ angle variable.

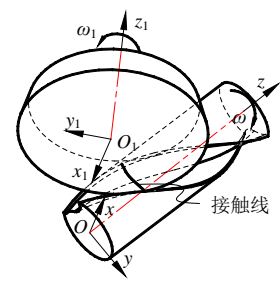

a The relative motion of a grinding wheel and workpiece
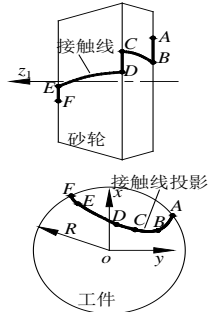

b Grinding wheel contact points

Fig 2 Analysis of contact line in groove machining

Expression of grinding wheel's recurrent surface under the fixed coordinates $\sigma$ is defined as follows:

$$
r=\left[\begin{array}{l}
x_{1} \cos \alpha-f\left(x_{1}\right) \sin \alpha \sin \varphi \\
a-f\left(x_{1}\right) \cos \varphi \\
-x_{1} \sin \alpha-f\left(x_{1}\right) \cdot \cos \alpha \sin \varphi
\end{array}\right]^{T}
$$

Fig 3(a) shows that change angle in the wheel and groove of the point of contact.

$$
\varphi=\sin ^{-1}\left(-L / \sqrt{M^{2}+N^{2}}\right)-\tan ^{-1}(M / N)
$$

In which $M=\left[x_{1}+f^{\prime}\left(x_{1}\right)\right] \tan \alpha, \quad N=(a+p \tan \alpha), \quad L=(p-a \tan \alpha) f^{\prime}\left(x_{1}\right)$ 。

In order to determine contact line of the grinding wheel bus inflection point, a new vector function is followed as (fig 3(b)):

$$
n_{C D}=\zeta \cdot n_{2 C}+(1-\zeta) \cdot n_{3 D}, 0 \leq \zeta \leq 1 。
$$

The formula ( 5 with substitution vector (4) solving the contact equation, the rotation angle is followed as:

$$
\varphi_{C D}=\zeta \cdot \varphi_{C}+(1-\zeta) \cdot \varphi_{D}
$$




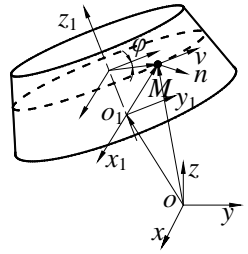

(a)contact points of rotary surface

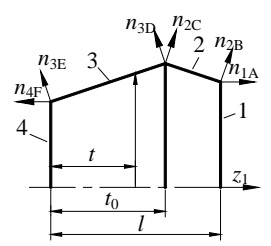

(b) vector of grinding wheel of inflection point

Fig. 3 Calculation of contact point

\subsection{Spiral groove theory model of wheel envelope into the exhibition}

The line of contact condition can be obtained by function (4) 、 function(6) and function(3).The contact line around the $\mathrm{X}$ axis as the spiral movement, the spiral groove surface equation can be get:ten:

$$
\left\{\begin{aligned}
x= & x_{1} \cos \alpha-f\left(x_{1}\right) \sin \alpha \sin \varphi+p \psi \\
y= & {\left[a-f\left(x_{1}\right) \cos \varphi\right] \cos \psi } \\
& +\left[x_{1} \sin \alpha+f\left(x_{1}\right) \cdot \cos \alpha \sin \varphi\right] \sin \psi \\
z= & {\left[a-f\left(x_{1}\right) \cos \varphi\right] \sin \psi } \\
& -\left[x_{1} \sin \alpha+f\left(x_{1}\right) \cdot \cos \alpha \sin \varphi\right] \cos \psi 。
\end{aligned}\right.
$$

$x_{1}, \varphi$ and $\psi$ are as variable, $x_{1}$ and $\varphi$ are satisfied function(7).

To $x=0$, the cross section of the helical can be obtained by formula (7).

\section{Examples}

\section{Grinding wheel shape and simulation example}

Grinding of micro milling cutter groove, a area between the wheel and groove is relatively small, using simple shape can guarante the shape precision of grinding wheel. In fig.4 (a) the diameter of the grinding wheel is $w$, coneangle and the thickness of the grinding wheel are respectively $\phi_{1}$ and $t_{1}$, chamfer and the thickness of chamfer are respectively $\phi_{2}$ and $t_{2}$. When $\mathrm{t} 2=0, \phi 1=\pi / 10, \phi 2=0, \alpha=\pi / 8$, the trench truncate is obtained by simulation calculation as shown in fig 2(b) .

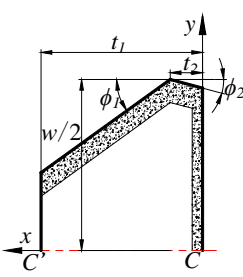

(a) cross-sectional shape of grinding wheel

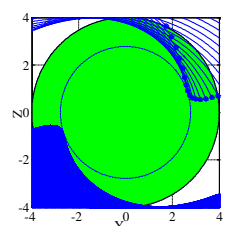

(b) simulation

Fig 4 grinding wheel and screw groove truncate simulation

\section{Grinding of spiral groove of micro vertical milling cutter}

\subsection{Bending analysis of groove grinding of micro vertical milling cutter milling}

A slender workpiece with low-rigidity is easy to occur deformation under the vertical press ure and becomes the formation of inverted cone or drum shaped structure. When the concentr ation stress of the micro milling cutter with inverted cone increases, its strength reduces. So $t$ he grinding of micro milling cutter, must be appropriate for the radial compensation, which can improve the inverted cone structure. During grinding, milling cutter rotating speed is low and can be considered as a static load by cantilever $F$ beam, the deflection can be expressed as:

$$
\delta(x)=\frac{F}{6 E I} x^{3}-\frac{F \cdot l}{2 E I} x^{2}+C_{1} \cdot x+C_{2}
$$

$l$ is the length of the cantilever slender rod, $E$ and $I$ respectively represent the elastic modulus and moment of inertia, $C_{1}$ and $C_{2}$ are constant.

Figure 5 (b) shows that the motion of the deflection adjustment grinding wheel in the direction of $\mathrm{Z}$ axis can effectively improve the inverted cone structure. 
Using the bar with diameter $0.11 \mathrm{~mm}$ in Figure 6,section shape can be obtained respectively by direct grinding and deflection compensation grinding way, which are shown in figure 7 .

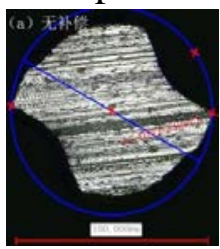

(a) Without compensation after

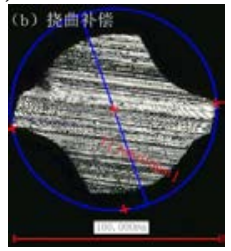

(b) Deflection compensation

Fig $7 \quad \phi 0.1 \mathrm{~mm}$ spiral groove truncate

Comparison of grinding results, without compensated directly grinding income trench truncat e chip groove diameter and flank groove diameter is too large, and according to the compensat ed truncate and Simulation of deflection calculation result is more consistent, that the compensat ion can effectively improve the net structure. Grinding temperature has great influence on the e nvironment shaped groove cut, the grinding experiment is obtained in the external temperature st able situation, through repeated grinding, truncate the obtained very stable,the average value of many measurements is shown in table 1 .

Table $1 \phi 0.1 \mathrm{~mm}$ groove geometry parameter value and the measured value

\begin{tabular}{cccc}
\hline Name & design value & $\begin{array}{c}\text { Before } \\
\text { compensation }\end{array}$ & after compensation \\
\hline $\begin{array}{c}\text { Front angle }{ }^{\circ} \\
\begin{array}{c}\text { The chip groove core } \\
\text { thickness } / \mu m\end{array}\end{array}$ & -6 & -6.35 & -6.22 \\
$\begin{array}{c}\text { Flank groove core } \\
\text { thickness } / \text { um }\end{array}$ & 75 & 75.35 & 75.06 \\
\hline
\end{tabular}

\section{Conclusion}

This paper studies the problem of calculation of grinding wheel movement envelope show spiral groove, according to contact theory, puts forward a method of calculating the grinding wheel and the spiral groove complete contact line.First of all, according to relative motion between grinding wheel and workpiece, establishes the coordinate transformation relationship between grinding wheel and workpiece, analysis the vector changes of the grinding wheel surface at the intersection to obtain the complete computational model of contact line.

During micro milling cutter processes grinding spiral groove in the edge, the paper puts forward the method to compensate the deflection using $\mathrm{Z}$ axis interpolation method.The method only needs the workpiece radial force obtained every grinding wheel feed.Experiments show that front angle and the core thickness with compensation monthly fine cutter is good in agreement with the milling spiral groove geometry parameters in simulation.

\section{Reference}

[1] H. Ohmori, K. Katahira, T. Naruse, et al. Microscopic Grinding Effects on Fabrication of Ultra-fine Micro Tools[J]. Annals of the CIRP, 2007, 56(1):569-572.

[2] H. Onikural, O. Ohnishi, Y. Take. Fabrication of Micro Carbide Tools by Ultrasonic Vibration Grinding[J]. Annals of the CIRP, 2000, 48(1):257-260.

[3] Osamu Ohnishi, H. Onikura, Seung Ki Min, et al. Characteristics of grooving by micro end mills with various tool shapes and approach to their optimal shape[J].Memoirs of the Faculty of Engineering, Kyushu University, 2007, 67(4):143-151.

[4] Peiyuan Li, J. A. J. Oosterling, A. M. Hoogstrate, et al. Design of micro square endmills for hard milling applications [J]. Int J Adv Manuf Technol, 2011, 57(9-12):859-870.

[5] S.K. KANG, K.F. Ehmann, C. Lin. A CAD approach to helical groove machining. Part 2 : numerical evaluation and sensitivity analysis[J]. International Journal of Machine Tools \& Manufacture, 1997, 37(1):101-117. 
[6] Yong Hyun Kim, S.L. Ko. Development of design and man- ufacturing technology for end mills in machining hardened steel[J]. Journal of Materials Processing Technology, 2002, 130-131:653-661.

[7] V. H. Nguyen, S. L. Ko. Determination of workpiece profile and influence of singular point in helical grooving[J]. Annals of the CIRP, 2013, 62(1):323-326.

[8] E. Uhlmann, C. Hübert. Tool grinding of end mill cutting tools made from high performance ceramics and cemented carbides[J]. Annals of the CIRP, 2011, 60(1):359-362. 